\title{
Neuroimmune Imbalance: The Key for the Treatment of Anxiety?
}

\author{
Bai Li, Tangxin Gao, Jing Du*
}

School of Medicine, Yunnan University, 2 Cuihu North Road, Kunming, 650091, Yunnan, China

Article Info

\section{Article Notes}

Received: May 30, 2019

Accepted: July 5, 2019

\section{${ }^{*}$ Correspondence:}

Dr. Jing Du, School of Medicine, Yunnan University, 2 Cuihu North Road, Kunming, 650091, Yunnan, China; Email: dujing@ynu.edu.cn.

(c) $2019 \mathrm{Du}$ J. This article is distributed under the terms of the Creative Commons Attribution 4.0 International License.
Anxiety disorder, a common mood disorder, seems associated with neuro-immune aberration on the pathophysiology, revealed by many clinical and preclinical evidences ${ }^{1-5}$. Furthermore, previous research suggested that the prefrontal cortex (PFC) is a critical brain region involved in anxiety disorders ${ }^{6-9}$. Now, we show that to keep the balance of pro-inflammatory immune-status and the anti-inflammatory immune-status in the PFC is the key for the treatment of anxiety ${ }^{10}$.

3'-deoxyadenosine (3'-dA), one of the major bioactive metabolites in the Cordyceps Militaris, possesses multiple beneficial effects of anti-inflammatory, anti-cancer, anti-viral, and anti-fungal activities ${ }^{11}$, 12. In recent study, we found that 3'-dA treatments significantly increased IL-4 expression in neurons, and IL-4 expression strongly correlated with the parameters representing reduced anxiety behaviours, suggesting that expression of the key anti-inflammatory molecule IL-4 is critical for the anxiolytic effect ${ }^{10}$. In addition, both 3 '- $\mathrm{dA}$ and traditional anxiolytic imipramine enhanced the expression levels of IL-4 and IL-10, and decreased the expression levels of IL-1 $\beta$ and TNF- $\alpha$, synchronous with the anxiolytic effects produced after chronic treatment for 5 days. The blockade of IL-4 action using the specific inhibitor RIL- $4 \mathrm{R} \alpha$ prevented the anxiolytic effect of both 3 '- $\mathrm{dA}$ and imipramine, indicating that correction of imbalance of proinflammatory and anti-inflammatory pathways is the common mechanism for the treatment of anxiety disorders ${ }^{10}$. Other anxiolytics, including diazepam, oryzanol, and chlordiazepoxide were shown enhancing anti-inflammatory cytokines and inhibiting pro-inflammatory cytokines in support of the findings ${ }^{13-16}$.

It is well established that pro-inflammatory cytokines, such as interleukin (IL)-6, TNF- $\alpha$ and IL-1 $\beta$, are increased in patients with anxiety disorders ${ }^{1}$. Conversely, the anti-inflammatory cytokine IL-4 is negatively associated with trait anxiety in adolescent girls ${ }^{2}$ and with anxiety symptoms in women in mid-pregnancy ${ }^{2,3}$. In an animal model, IL-4 was reduced in anxious mice ${ }^{4}$, and an IL-4 injection attenuated anxiety-like behaviour ${ }^{5}$ and a previous study demonstrated that IL-4-knockout mice exhibited anxiety-like behaviour ${ }^{18}$. Immobilization stress leads to decreased release of IL-4 from the neurons of the locus coeruleus ${ }^{4}$. Growing evidence indicates the balance between pro-inflammatory and anti-inflammatory factors is associated with the pathophysiology of anxiety disorder ${ }^{17}$. Increased inflammatory reactions in the CNS are commonly seen in patients with anxiety disorders.

3 '- $\mathrm{dA}$ was reported to be able to modulate peripheral immune functions, Downregulating the gene expression of the proinflammatory factors IL-1 $\beta$, TNF- $\alpha$, and IL-2 and upregulating the 
anti-inflammatory factors IL-4, IL-10, IL-1R $\alpha$, and TGF- $\beta$ in macrophages and mononuclear cells in the peripheral immune system $^{18-20}$, similar to what happened in CNS. The lack of IL-4, a key molecule to modulate neuroimmune status, leads to leads to increased neuroinflammation in response to lipopolysaccharide in mice $^{21}$. The action of IL-4 results from its ability to inhibit the release of proinflammatory cytokines, such as IL- $1 \beta$ and TNF- $\alpha$, by innate immune cells and to upregulate the synthesis of antiinflammatory factors, such as IL- $1 \beta$ receptor antagonist ${ }^{19}$, ${ }^{22}$. In addition, neuronal IL-4 plays a vital role in brain cleanup and repair after ischemic damage ${ }^{23}$. Endogenous IL-4 is expressed mainly in neurons, although small amounts co-localize with GFAP-positive cells and Iba1-immunoreactive cells ${ }^{4}$. Our study shows that 3 '-dA exhibited a rapid and stable anxiolytic effect in the mice behavioural tests via activating the IL-4 pathway, regulating the expression levels of the IL-10 IL-1 $\beta$ and TNF- $\alpha$ in PFC, ${ }^{10}$, suggesting 3 '-dA functions to correct the imbalance between proinflammatory factors and anti-inflammatory factors, which is helpful for restraining inflammatory reactions, activating anti-inflammatory factor pathways, and promoting neuralfunctional repair in the CNS.

Furthermore, cumulative studies show that transition of pro-inflammatory to anti-inflammatory status helps to modulate neuronal and synaptic functions. It was previously reported that restoration of IL-4 concentrations in the hippocampus to the levels of those observed in young rats is associated with the successful maintenance of long-term potentiation (LTP), and the downregulation of IL-4-stimulated signalling significantly contributes to the deficit in LTP ${ }^{19,24,25}$. LTP is an important indicator of neural synaptic plasticity, which plays a key role in behavioural manifestations ${ }^{26,27}$, and may be a relevant mechanism in IL-4-mediated anxiolytic effects of 3'-dA and imipramine.

In summary, both 3'-dA and the traditional antianxietic imipramine produced an anxiolytic effect via increasing the IL-4 expression levels to correct the pro-inflammatory/ anti-inflammatory balance, subsequently to change the neuronal functions and behaviours, and the IL-4 specific inhibitor RIL-4R $\alpha$ could prevented the anxiolytic effect of both 3'-dA and imipramine. These results indicate that IL-4 pathway is the key to the treatment of anxiety disorder and provide an innovative drug with a novel neuro-immune mechanism for the treatment of anxiety disorder.

\section{References}

1. Quagliato LA, Nardi AE. Cytokine alterations in panic disorder: A systematic review. J Affect Disord. 2018; 228: p. 91-96.

2. Dorn LD, Gayles JG, Engeland CG, et al. Cytokine Patterns in Healthy Adolescent Girls: Heterogeneity Captured by Variable and PersonCentered Statistical Strategies. Psychosom Med. 2016; 78(6): p. 646-56.

3. Karlsson L, Nousiainen N, Scheinin NM, et al. Cytokine profile and maternal depression and anxiety symptoms in mid-pregnancy-the
FinnBrain Birth Cohort Study. Arch Womens Ment Health. 2017; 20(1): p. 39-48.

4. Lee HJ, Park HJ, Starkweather A, et al. Decreased Interleukin-4 Release from the Neurons of the Locus Coeruleus in Response to Immobilization Stress. Mediators Inflamm. 2016; 2016: p. 3501905.

5. Han A, Yeo H, Park MJ, et al. IL-4/10 prevents stress vulnerability following imipramine discontinuation. J Neuroinflammation. 2015; 12: p. 197.

6. Tovote P, Fadok JP, Lüthi A. Luthi, Neuronal circuits for fear and anxiety. Nat Rev Neurosci. 2015; 16(6): p. 317-31.

7. Likhtik E, Stujenske JM, Topiwala MA, et al. Prefrontal entrainment of amygdala activity signals safety in learned fear and innate anxiety. Nat Neurosci. 2014; 17(1): p. 106-13.

8. Birn RM, Shackman AJ, Oler JA, et al. Evolutionarily conserved prefrontal-amygdalar dysfunction in early-life anxiety. Mol Psychiatry. 2014; 19(8): p. 915-22.

9. Adhikari A1, Topiwala MA, Gordon JA. Synchronized activity between the ventral hippocampus and the medial prefrontal cortex during anxiety. Neuron. 2010; 65(2): p. 257-69.

10. Gao T, Li B, Hou Y, et al. Interleukin-4 signalling pathway underlies the anxiolytic effect induced by 3-deoxyadenosine. Psychopharmacology (Berl). 2019.

11. Qin P, Li X, Yang H, et al. Therapeutic Potential and Biological Applications of Cordycepin and Metabolic Mechanisms in CordycepinProducing Fungi. Molecules. 2019; 24(12).

12. Tuli HS, Sharma AK, Sandhu SS, et al. Cordycepin: a bioactive metabolite with therapeutic potential. Life Sci. 2013; 93(23): p. 863-9.

13. Obuchowicz E, Bielecka AM, Paul-Samojedny M, et al. Imipramine and fluoxetine inhibit LPS-induced activation and affect morphology of microglial cells in the rat glial culture. Pharmacol Rep, 2014; 66(1): p. 34-43.

14. Gomes EP1, Aguiar JC, Fonseca-Silva T, et al. Diazepam reverses the alveolar bone loss and hippocampal interleukin-1beta and interleukin- 6 enhanced by conditioned fear stress in ligatureinduced periodontal disease in rats. J Periodontal Res. 2013; 48(2): p. 151-8.

15. Rao YPC, Sugasini D, Lokesh BR. Dietary gamma oryzanol plays a significant role in the anti-inflammatory activity of rice bran oil by decreasing pro-inflammatory mediators secreted by peritoneal macrophages of rats. Biochem Biophys Res Commun. 2016; 479(4): p. 747-752.

16. Curtin NM, Mills KH, Connor TJ. Psychological stress increases expression of IL-10 and its homolog IL-19 via beta-adrenoceptor activation: reversal by the anxiolytic chlordiazepoxide. Brain Behav Immun. 2009; 23(3): p. 371-9.

17. Quagliato LA, Nardi AE. Cytokine alterations in panic disorder: A systematic review. J Affect Disord. 2017; 228: p. 91-96.

18. Zhou X, Meyer CU, Schmidtke P, et al. Effect of cordycepin on interleukin-10 production of human peripheral blood mononuclear cells. Eur J Pharmacol. 2002; 453(2-3): p. 309-17.

19. Nolan Y, Maher FO, Martin DS, et al. Role of interleukin-4 in regulation of age-related inflammatory changes in the hippocampus. J Biol Chem. 2005; 280(10): p. 9354-62.

20. Seo MJ, Kim MJ, Lee HH, et al. Effect of cordycepin on the expression of the inflammatory cytokines TNF-alpha, IL-6, and IL-17A in C57BL/6 mice. J Microbiol Biotechnol. 2013; 23(2): p. 156-60.

21. Lyons A, McQuillan K, Deighan BF, et al. Decreased neuronal CD200 expression in IL-4-deficient mice results in increased neuroinflammation in response to lipopolysaccharide. Brain Behav Immun. 2009; 23(7): p. 1020-7. 
22. Lyons A, Griffin RJ, Costelloe CE, et al. IL-4 attenuates the neuroinflammation induced by amyloid-beta in vivo and in vitro. Neurochem. 2007; 101(3): p. 771-81.

23. Zhao X, Wang $H$, Sun G, et al. Neuronal Interleukin-4 as a Modulator of Microglial Pathways and Ischemic Brain Damage. J Neurosci. 2015; 35(32): p. 11281-91.

24. Maher FO, Nolan Y, Lynch MA. Downregulation of IL-4-induced signalling in hippocampus contributes to deficits in LTP in the aged rat. Neurobiol Aging. 2005; 26(5): p. 717-28.
25. Clarke RM, Lyons A, O'Connell F, et al. A pivotal role for interleukin-4 in atorvastatin-associated neuroprotection in rat brain. J Biol Chem. 2008; 283(4): p. 1808-17.

26. Huganir RL, Nicoll RA. AMPARs and synaptic plasticity: the last 25 years. Neuron. 2013; 80(3): p. 704-17.

27. He Y, Kulasiri D, Samarasinghe S. Modelling bidirectional modulations in synaptic plasticity: A biochemical pathway model to understand the emergence of long term potentiation (LTP) and long term depression (LTD). J Theor Biol. 2016; 403: p. 159-177. 